\title{
AUTOSOSTENIBILIDAD DEL SISTEMA DE TRANSPORTE PÚBLICO MASIVO TRANSMILENIO S.A.
}

Giselle Juliette Alvarez Polanco

Facultad de Negocios, Gestión y Sostenibilidad

Especialización en Gestión Empresarial

Especialista en Gestión Empresarial

gisili53@ hotmail.com

Cesar Iván Fernández Cárdenas

Facultad de Negocios, Gestión y Sostenibilidad

Especialización en Gestión Empresarial

Especialista en Gestión Empresarial cesarfernandezcar@gmail.com

Franf Helver Garzón Lozano

Facultad de Negocios, Gestión y Sostenibilidad Especialización en Gestión Empresarial Especialista en Gestión Empresarial franfhelver83@hotmail.com 
Roberto José Madrid Gutiérrez

Facultad de Negocios, Gestión y Sostenibilidad

Especialización en Gestión Empresarial

Especialista en Gestión Empresarial

rjmadridg@gmail.com

\section{Resumen}

"El presente artículo trata el siguiente problema ¿Cómo lograr una operación sostenible de la empresa de transporte masivo TRANSMILENIO S.A.?, para este propósito se utilizó el método de la prospectiva estratégica desarrollado por Michel Godet,1987;

donde se identificaron las principales variables de cambio, las cuales fueron clasificadas a través de un análisis estructural, que permitió identificar en la zona de poder a la Gestión Política y el Cumplimiento Implementación del PIGA, como las variables estratégicas que debe administrar la empresa.

Palabras clave: Prospectiva, Variables, Transporte Masivo, Estratégico” 
Introducción

La sostenibilidad del Sistema De Transporte Público Masivo TRANSMILENIO S.A., en Bogotá no es un concepto que tenga un solo sentido. Puede entenderse de diferentes maneras. Desde un enfoque amplio abarca los factores social, ecológico, económico, político entre otros. Esta significa que los recursos asignados deben ser suficientes para cubrir, después de transferencias, los costos de operación y de inversión del sistema, la autosostenibilidad financiera debe ser compatible con la autosostenibilidad social. Donde también se debe tener en cuenta los factores ecológicos y políticos que la rodean.

Actualmente Transmilenio S.A cuenta con 114,4 Kms de cobertura en la ciudad de Bogotá, nueve portales, 139 estaciones regulares, 12 corredores en servicio y funciona con el desarrollo de III fases las cuales están articulas por medio de 11 diferentes operadores, diariamente en promedio moviliza a 2.253.082 de usuarios lo que en ocasiones hace que sus rutas no sean tan frecuentes como estos desearían, la inseguridad y falta de cultura ciudadana hacen que Transmilenio S.A no sea el mejor Sistema integrado de transporte que desearía y necesita la ciudad de Bogotá (Transmilenio S.A., 2018).

Se pretende analizar los posibles factores que inciden negativamente para que la autosostenibilidad de Transmilenio no sea algo fácil de lograr y que el sistema de transporte además de integrado debe ser eficiente, eficaz y satisfactorio para toda sociedad, que finalmente es quien utiliza el servicio y paga por él. Es lograr identificar posibles estrategias que permitan a Transmilenio plantear soluciones tanto en su estructura de movilidad como en su estructura financiera para su sostenimiento como empresa y como prestadora de un servicio social.

La metodología utilizada para el análisis de las variables y factores que intervienen en el problema fueron analizadas por medio de un análisis estructural ; Esta metodología está compuesta por varias etapas, donde la primera consiste en identificar factores de cambio, la cual consiste en identificar las principales variables que en el futuro tendrán un 
impacto en la empresa; la segunda consiste en una clasificación de variables, que permita identificarlas y clasificarlas de mayor a menor orden de importancia; finalmente se procede a un análisis de impactos cruzados, que se desarrolla a través de una matriz que permite evaluar tanto la influencia como la dependencia que existe entre las diferentes variables.

\section{Marco Teórico}

Hace 20 años, cuando se empezó a implementar la estrategia de transporte del tercer milenio, Bogotá contaba con una demanda potencial de 6 millones de habitantes, que se proyectó atender con una infraestructura planeada en total nueve etapas, llamadas 'fases'. En la actualidad, solo se han construido tres fases, mientras que, de forma paralela, la demanda potencial de usuarios siguió creciendo, situándose a le fecha en cerca de 8 millones de usuarios; quienes, en su vida urbana, tienen derecho a la movilidad, es decir el libre desplazamiento en condiciones óptimas de relación entre medio ambiente, espacio público e infraestructura (Ballén, 2007).

Según (Jolonch, 2013), en la ciudad no se dio una planeación lógica en sus arterias viales, con en obras inconclusas, vías en estados deplorables, corrupción, cambio continuo de altos funcionarios de obras públicas; donde se sumó la necesidad de atender la mayor cantidad de usuarios del sistema, a través de una planeación de rutas, que pretendía incluir todas las zonas de importancia cómo puerto de usuarios en la ciudad; sin prever, que esta planeación resulto contraproducente para los usuarios, debido al número excesivo de las mismas y la falta de precisión en la prestación del servicio, que se conjugan para tornarse complejas de entender por parte del usuario

Aspectos como la sincronización, espacio y velocidad son propios en el diseño y planeación de rutas; sin embargo, hay aspectos que ocurren en el sistema real, de tipo idiosincrático, algunos propios de la cultura bogotana que están incidiendo en el sistema (Rodríguez, 2014); donde los altercados entre choferes y pasajeros, usuarios con el ceño

Fruncido, violación de mujeres, agresión a niños, accidentes de tránsito y retrasos, se han mal interpretado como la cultura ciudadana en el sistema, la cual repercute en la calidad del servicio y en la mala imagen de la empresa en las calles. 
A diferencia del transporte, la movilidad aborda no solo infraestructura y vehículos, sino que incorpora condiciones sociales, políticas, económicas y culturales de quienes se movilizan (Asher, 2004). Los resultados del estudio adelantado por (Velásquez, 2009), muestran una relación directamente proporcional entre la demanda del sistema, y el número de estaciones, la población, el PIB per cápita de la ciudad, siendo este último, uno de los elementos claves al momento de estudiar las necesidades de actualización de tarifas, como un requisito para la autosostenibilidad presupuestal de Transmilenio.

De lo anterior, se desprende un desafío importante que consiste en diseñar una política pública de movilidad y, en consecuencia, una visión más técnica reflejada en la primacía de los modos de transporte, las vías públicas, las infraestructuras y las condiciones económicas de la población; generando la idea de que estos son medios o instrumentos al servicio de la movilidad de los ciudadanos y no un fin en sí mismos (Gibsone, 2011). También es importante vincular en esta propuesta de política pública, el "enfoque de oferta" como un marco para el diseño y la implementación de la verdadera movilidad que requiere la ciudad de Bogotá, donde prime la perspectiva del desarrollo humano y el respeto por el medio ambiente, en oposición al tradicional "enfoque de demanda" que solo se centra en aspectos financieros.

Finalmente se quiere indicar, que Transmilenio es un sistema cuya infraestructura (vías y estaciones), se desarrolla con recursos públicos, y la administración, planificación y organización del sistema la hace una empresa pública, TRANSMILENIO S.A. sin embargo, los vehículos son propiedad privada, el recaudo lo hace otra empresa privada (ANGELCOM), y el distrito de

Bogotá recibe el $3 \%$ de las utilidades, con las que debe mantener la infraestructura del sistema, mientras los transportadores se reparten el resto.

Metodología

Se empleó la metodología del análisis estructural, que fue desarrollada por el académico francés Michel Godet en el año de 1987. Esta metodología está compuesta por 
varias etapas, donde la primera consiste en identificar factores de cambio, la cual consiste en identificar las principales variables que en el futuro tendrán un impacto en la empresa; la segunda consiste en una clasificación de variables, que permita identificarlas y clasificarlas de mayor a menor orden de importancia; finalmente se procede a un análisis de impactos cruzados, que se desarrolla a través de una matriz que permite evaluar tanto la influencia como la dependencia que existe entre las diferentes variables.

De acuerdo a (Godet, 1993), las etapas de la metodología anteriormente descrita se desarrollaron en un aplicativo implementado en una hoja de cálculo de Excel®, donde se siguió el siguiente procedimiento:

1) Listar todas las variables identificadas en la pestaña de tendencias, donde se hace una descripción y se les define un indicador.

2) Clasificación de las variables, identificando el tipo de factor en que se encuentran circunscritas, que permita posteriormente seleccionar el cuadrante de desempeño al interior de una matriz DOFA.

3) Evaluación del impacto de cada variable, a través de una escala de cero (0) a tres (3), donde este último representa un alto impacto.

4) Determinación del orden de importancia de las variables, el cual se desarrolla a través de una clasificación objetiva de las variables, ordenándolas de mayor a menor nivel de importancia, de acuerdo al resultado de la evaluación de impactos.

5) Evaluación de impactos cruzados, que consiste en evaluar tanto el nivel de dependencia como de influencia que existe entre las variables, a través de una escala de cero (0) a tres (3), donde este último representa un alto impacto.

6) Generación del gráfico de subsistemas, el cual genera de forma automática el aplicativo en la pestaña denominada MIC-MAC.

Una vez se concluye la evaluación de impactos cruzados, el aplicativo genera un gráfico sobre esta matriz y la multiplicación aplicada a la clasificación de influencia y 
dependencia de las variables claves; que permitirá visualizar la clasificación de estas, cómo autónomas, de resultado, de conflicto o de poder dentro de cada una de las zonas del gráfico MIC-MAC (ver Gráfico 1).

\section{Resultados}

Gráfico 1: Representación gráfica MIC-MAC

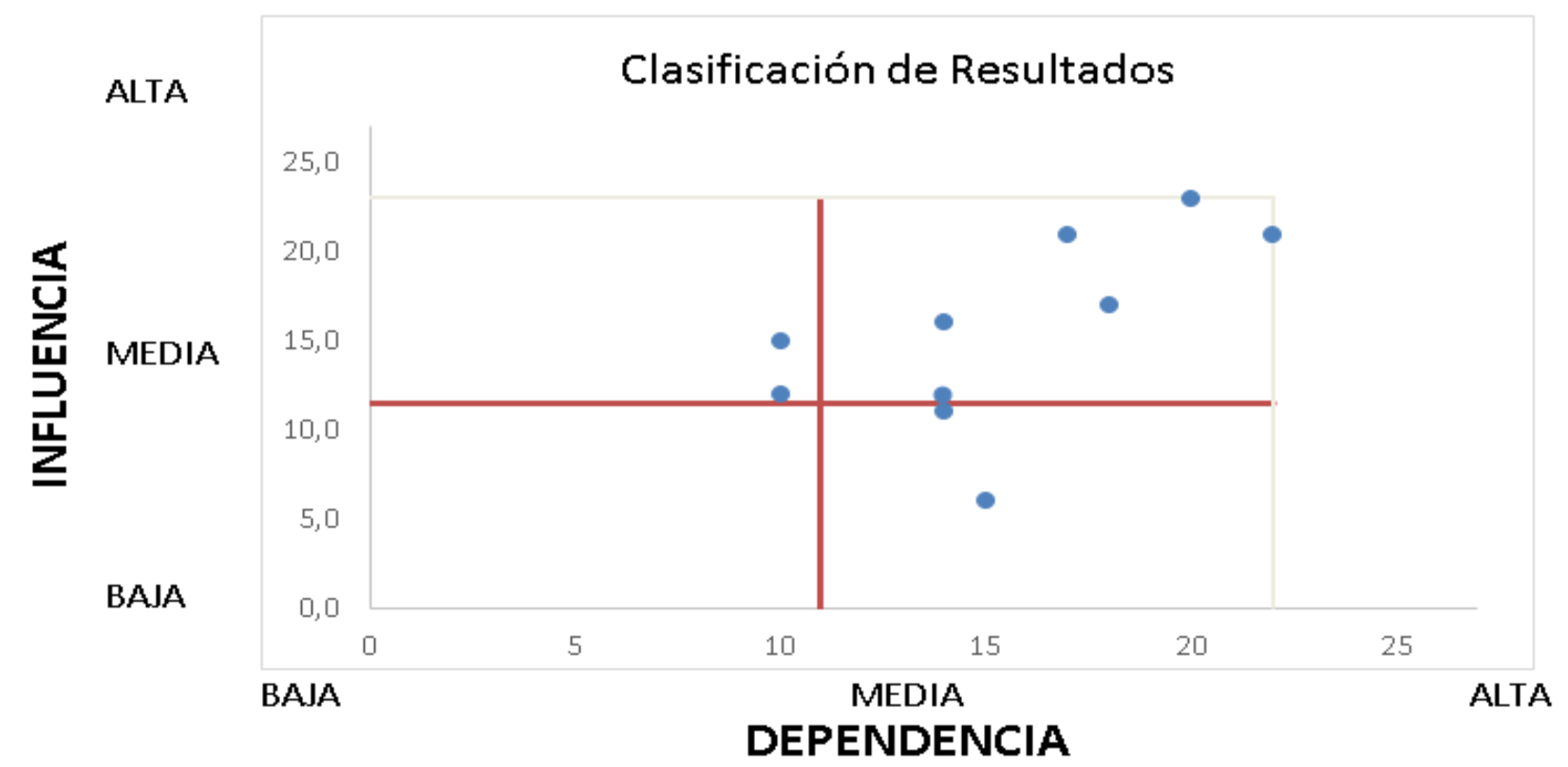

Fuente: Elaboración propia (2019)

\section{Análisis variables de poder y de conflicto:}

Variables de conflicto: De acuerdo con el ejercicio de prospectiva desarrollado, se identificaron las siguientes variables:

- Crecimiento de la demanda.

- Actualización de tarifas.

- Cultura ciudadana.

- Emisión de gases contaminantes.

- Autosostenibilidad presupuestal.

- Planeación de rutas. 
Desde el punto de vista estratégico, estas variables son las más dinámicas del sistema, y por lo tanto, sobre ellas hay que actuar de forma inmediata, porque están bajo el control del mismo. En consecuencia, hay que desarrollar estrategias de muy poco plazo para afectarlas, entre las estrategias más relevantes para cada una de las variables descritas encontramos:

Para atender el crecimiento de la demanda se debe revisar la viabilidad de aumentar la capacidad de las estaciones, de acuerdo con los estudios específicos realizados, se recomienda trasladar las actuales taquillas fuera de las estaciones y evaluar la implementación de segundos accesos en las estaciones de los corredores que aún no las tienen.

Por otra parte, para la autosostenibilidad presupuestal se debe hacer seguimiento a la ejecución presupuestal de la entidad periódicamente, así mismo se sugiere revisar los recursos asignados al Fondo de Estabilidad Tarifaria en donde se encuentra la mayor necesidad de recursos actualmente, debido a la tarifa actual del sistema.

Para la planeación de las rutas se debe regular y controlar los tiempos de frecuencia de los servicios troncales, optimizando el tránsito en las zonas críticas, así mismo se debe diseñar una campaña complementaria para generar una cultura del usuario que permita una mejor operación y recuperación de la capacidad del sistema.

Variables de poder: De acuerdo con el ejercicio de prospectiva desarrollado, se identificaron las siguientes variables:

- Gestión política.

- Cumplimiento político del PIGA.

Aunque estas variables no están bajo el control del sistema, de todas formas inciden de forma muy influyente. En consecuencia, hay que desarrollar algunas estrategias de mediano plazo y largo plazo para afectarlas, entre las estrategias más relevantes para cada una de las variables descritas encontramos: 
El Plan Institucional de Gestión Ambiental es un instrumento de planeación que parte del análisis de la situación ambiental de TRANSMILENIO S.A., con el propósito de brindar información y argumentos necesarios para el planteamiento de acciones de gestión ambiental que garanticen primordialmente el cumplimiento de los objetivos de ecoeficiencia establecidos en el Decreto 456 de 2008, entre otras acciones ambientales que contemple la Entidad y aporten a la totalidad de los objetivos ambientales establecidos en el PGA. De esta manera se pretende avanzar hacia la adopción e implementación de un sistema integrado de gestión, que en materia ambiental, se basan en la norma técnica NTCISO 14001; y que se debe realizar de manera gradual conforme a la evolución de la entidad.

Para el cumplimiento de la política de PIGA se debe acatar en su totalidad el Plan Institucional de Gestión Ambiental de acuerdo con lo dispuesto por la Ley y evidenciar el impacto ambiental en la Ciudad por el cumplimiento de la gestión ambiental de forma efectiva, se recomienda adelantar las actividades programadas en el Plan de Acción PIGA obteniendo un promedio anual de avance de ejecución cada vez mayor y revisar las actividades que no tuvieron un avance total.

Este Plan tiene 3 elementos principales:

La Gestión ambiental institucional: Contempla los procesos de gestión ambiental que se realizan en las áreas que administra TRANSMILENIO S.A. (sede administrativa, portales y estaciones)

La Gestión ambiental del Sistema: Se relaciona con la gestión ambiental del Sistema y el seguimiento y control a la gestión que realizan las empresas operadoras del Sistema en el marco de los contratos de concesión.

Coordinación Interinstitucional: Contempla los procesos, mecanismos de comunicación y concertación y las interrelaciones que se establecen con las demás entidades del Distrito y con universidades, organismos y entidades de orden nacional e internacional que se relacionan con el sistema (Cartilla sistema integrado de gestión de calidad, 2018). 
La Gestión Política relacionada con TRANSMILENIO S.A., actualmente es coherente con las normas vigentes, el plan de movilidad actual, las necesidades de los usuarios y partes interesadas, así como las funciones asignadas a la Entidad y los programas y proyectos que esta desarrolle, en busca de mejorar la calidad de vida de los ciudadanos, planeando, gestionando y controlando el desarrollo del Sistema Integrado de Transporte Público de pasajeros en la ciudad de Bogotá, en cumplimiento de los requisitos legales y organizacionales.

De igual manera, la gestión política está relacionada con el desarrollo y mejoramiento de la infraestructura del sistema, para lograr esta finalidad se sugiere adecuar la infraestructura actual, para acomodar la demanda creciente y poder brindar un servicio de mayor capacidad. Esta estrategia permitirá acomodar servicios de alta capacidad basados en una flota mayor de biarticulados, el diseño de megaestaciones o portales intermedios.

Esta estrategia se compromete con:

La satisfacción de las partes interesadas.

La identificación, mitigación y tratamiento de los riesgos institucionales.

La prevención de la contaminación y la mitigación de los impactos ambientales.

La identificación y la prevención de las condiciones y factores que afectan o pueden afectar la salud y seguridad del personal, para garantizar un ambiente de trabajo adecuado.

La protección de la confidencialidad, integridad, disponibilidad y autenticidad de los activos de información (El Tiempo, 2014).

Con esto se busca fortalecer el desarrollo de los trabajadores de la Entidad y la participación ciudadana, promoviendo la sostenibilidad del Sistema Integrado de Gestión y su mejora continua. 


\section{Análisis riesgos potenciales y propuestas para mitigar el impacto de los mismos}

A continuación, se hace una descripción de los riesgos potenciales a los que se enfrenta la empresa y de las estrategias propuestas para mitigar el impacto del mismo:

Tabla 1: Riesgos potenciales y estrategias para mitigarlos.

\begin{tabular}{|c|c|c|}
\hline $\begin{array}{l}\text { VARIABLES DE } \\
\text { CONFLICTO }\end{array}$ & RIESGO & ESTRATEGIA \\
\hline Emisión de gases contaminantes. & $\begin{array}{l}\text { Direccionamiento de los } \\
\text { conceptos de carácter } \\
\text { ambiental para la adjudicación } \\
\text { de contratos, que favorezcan } \\
\text { un interés personal o } \\
\text { particular. }\end{array}$ & $\begin{array}{lrr}\text { Diseñar, } & \text { desarrollar, } \\
\text { coordinar y } & \text { realizar } \\
\text { seguimiento a } & \text { políticas, } \\
\text { programas, } & \text { proyectos, } \\
\text { investigaciones y actividades } \\
\text { de mitigación de } & \text { impactos } \\
\text { ambientales e intervención de } \\
\text { aspectos ambientales de los } \\
\text { contratos adjudicados. }\end{array}$ \\
\hline Cultura ciudadana. & $\begin{array}{l}\text { Intereses indebidos de la } \\
\text { Entidad que entrega } \\
\text { información falsa para su } \\
\text { divulgación por los canales } \\
\text { externos. }\end{array}$ & $\begin{array}{l}\text { Mantener informado a los } \\
\text { grupos de interés de } \\
\text { comunicación externa sobre la } \\
\text { gestión de la Entidad y el } \\
\text { estado del SITP con } \\
\text { información verdadera. }\end{array}$ \\
\hline Actualización de tarifas. & $\begin{array}{l}\text { Manipulación de la } \\
\text { información en relación con el } \\
\text { esquema tarifario para } \\
\text { beneficio de terceros. }\end{array}$ & $\begin{array}{l}\text { Determinar y formular el } \\
\text { esquema tarifario del Sistema } \\
\text { Integrado de Transporte } \\
\text { Público. }\end{array}$ \\
\hline
\end{tabular}




\begin{tabular}{|c|c|c|}
\hline Autosostenibilidad presupuestal. & $\begin{array}{l}\text { Manipular información } \\
\text { relacionada con los recursos } \\
\text { financieros de la entidad para } \\
\text { beneficio de un tercero o } \\
\text { propio. }\end{array}$ & $\begin{array}{l}\text { Asegurar el buen manejo y } \\
\text { control al recaudo del SITP a } \\
\text { través del recaudo de la tarifa. }\end{array}$ \\
\hline $\begin{array}{l}\text { Planeación de rutas. } \\
\text { Crecimiento de la demanda. }\end{array}$ & $\begin{array}{l}\text { Manipulación de los } \\
\text { parámetros de la } \\
\text { programación de rutas con el } \\
\text { fin de favorecer a terceros. }\end{array}$ & $\begin{array}{l}\text { Verificar el cumplimiento de } \\
\text { lo estipulado en los contratos } \\
\text { de concesión y en el manual } \\
\text { de operaciones del } \\
\text { componente troncal y } \\
\text { alimentador del SITP. }\end{array}$ \\
\hline
\end{tabular}

Fuente: Elaboración propia.(2019)

\section{Conclusiones}

El análisis estructural permitió identificar dos tendencias ubicadas en la zona de poder concentrada en la gestión política y el cumplimiento implementación del plan integral de gestión ambiental (PIGA), como las variables estratégicas que debe administrar la empresa.

La gestión política es una variable muy importante para el crecimiento de sistema y la sostenibilidad del mismo, pero esta crea una dependencia de alguna manera de la gestión de recursos públicos, pero crea una oportunidad de convertir el servicio de transporte público de los bogotanos de una política pública para la movilidad de la ciudad, esto aumenta las posibilidades de financiar nuevos proyectos para el SITP.

Se debe trabajar en la articulación de todos los departamentos de la empresa, comercial, comunicaciones, involucrados en la comunicación interna y externa, toda la estrategia diseñada debe pasar por todos los departamentos de la entidad, para lograr sincronización que coadyuve a construir cultura ciudadana, a través de nuevas estrategias cercanas al usuario. 
Atender el crecimiento de la demanda, incrementar capacidad de estaciones, además, trasladar las taquillas existentes, a zonas externas de las estaciones, junto con accesos en las estaciones en los corredores donde aún no la tienen.

Mayor control en los tiempos de frecuencia de los servicios en todas las troncales, esto ayudaría a mejorar la presencia en las zonzas de mayor demanda, es necesario diseñar a la par una estrategia con los ciudadanos que ayude a la operación del sistema y capacidad del mismo.

Se debe desarrollar y gestionar una política pública, que conduzca al desarrollo y mejoramiento de la infraestructura vial, que requiere el sistema en la ciudad y en los puntos más críticos.

Analizar y formular un nuevo esquema tarifario integrado al transporte público, más acorde a la realidad del sistema y de los clientes, al tiempo que debe crearse un mecanismo de buen recaudo del SITP.

La metodología compuesta de varias etapas, permite consolidar factores de cambios que más impactaran en el futuro a la empresa, anticipándonos más asertivamente de acuerdo al resultado de los impactos cruzados, de manera tal, que se convierte en una herramienta que puede predecir futuros escenarios y clasificar el nivel de importancia.

Definir una formula con un nuevo esquema tarifario integrado al transporte público, más acorde a la realidad del sistema y de los clientes, al tiempo que debe crearse un mecanismo de buen recaudo del SITP. 
Bibliografía

Asher, F. (2004). Los principios del nuevo urbanismo. Madrid: Alianza.

Ballén, F. (2007). Derecho a la movilidad. La experiencia de Bogotá D.C. Prolegómenos. Derechos y valores, X(20), 169-181.

Gibsone, C. D. (2011). Algunas reflexiones sobre la movilidad urbana en Colombia, desde la perspectiva del desarrollo humano. Papel Político, 16(2), 485-514.

Godet, M. (1993). De la anticipación a la acción. Barcelona, España: Marcombo.

Jolonch, J. (2013). Análisis del transporte masivo y la movilidad en Bogotá. Universidad \& Empresa, 24, 15-23.

Rodríguez, C. (2014). Modelamiento de estaciones Transmilenio mediante Automatas Celulares: Lecciones aprendidas. En: Ingeniería, 19(2), 105-113.

Transmilenio S.A. (Noviembre de 2018). Estadísticas de oferta y demanda del Sistema Integrado de Transporte Público -SITP. Obtenido de https://www.transmilenio.gov.co/publicaciones/151075/estadisticas-de-oferta-y-demandabimensual-del-sistema-integrado-de-transporte-publico---sitp---noviembre---diciembre--$2018 /$

Velásquez, J. M. (2009). Análisis de factores que inciden en la demanda del sistema Transmilenio en Bogotá. Revista de Ingenieria, (30), 56-64. 